\title{
Cryptanalysis of AFMAP
}

\author{
Masoumeh Safkhani $^{1 \mathrm{a})}$, Majid Naderi ${ }^{1 \mathrm{~b})}$, and Nasour Bagheri ${ }^{2 \mathrm{c})}$ \\ ${ }^{1}$ Security and Cryptography Laboratory, Electrical Engineering Department, \\ Iran University of Science and Technology \\ ${ }^{2}$ Electrica Engineering Department, Shahid Rajaee University, Tehran, Iran \\ a)m_safkhani@iust.ac.ir \\ b)m_naderi@iust.ac.ir \\ c)na.bagheri@gmail.com
}

Abstract: Radio frequency identification systems which use radio frequency for object identification are widely developed nowadays. Security problems such as privacy and untraceability had lead to propose numerous protocols for RFID systems. However most of these attempts have not been successful.

In this paper we present a desynchronization attack against a recently proposed RFID protocol called Anonymous Forward-Secure Mutual Authentication or in short AFMAP. The complexity of attack is a few runs of the protocol and the success probability is 1 . To the best of our knowledge, this is the first attack on AFMAP.

Keywords: RFID, Anonymous Forward-Secure Mutual Authentication Protocol, AFMAP, desynchronization

Classification: Science and engineering for electronics

\section{References}

[1] M. Bárász, B. Boros, P. Ligeti, K. Lója, and D. Nagy, "Passive attack against the m2ap mutual authentication protocol for rfid tags," First International EURASIP Workshop on RFID Technology, Sept. 2007.

[2] T. Cao, E. Bertino, and H. Lei, "Security analysis of the sasi protocol," IEEE Trans. Dependable Sec. Comput., vol. 6, no. 1, pp. 73-77, 2009.

[3] J. C. Hernandez-Castro, J. M. E. Tapiador, P. Peris-Lopez, and J.-J. Quisquater, "Cryptanalysis of the sasi ultralightweight rfid authentication protocol with modular rotations," Technical Report arXiv:0811.425\%, Nov. 2008.

[4] C. Hung-Yu, "SASI: A New Ultralightweight RFID Authentication Protocol Providing Strong Authentication and Strong Integrity," IEEE Trans. Dependable Sec. Comput., vol. 4, no. 4, pp. 337-340, Dec. 2007.

[5] T. Li and R. H. Deng, "Vulnerability analysis of emap - an efficient rfid mutual authentication protocol," Second International Conference on Availability, Reliability and Security - AReS, Vienna, Austria, April 2007.

[6] T. Li, G. Wang, and R. H. Deng, "Security analysis on a family of ultralightweight rfid authentication protocols," Journal of Software, vol. 3, no. 3, March 2008.

[7] P. Peris-Lopez, J. C. H. Castro, J. M. Estévez-Tapiador, and A. Ribagorda, "Advances in ultralightweight cryptography for low-cost rfid tags: Gossamer protocol," WISA, pp. 56-68, 2008. 
[8] P. Peris-Lopez, J. C. Hernandez-Castro, J. M. Estevez-Tapiador, and A. Ribagorda, "EMAP: An Efficient Mutual Authentication Protocol for Low-Cost RFID Tags," OTM Federated Conferences and Workshop: IS Workshop - IS'06, vol. 4277 of Lecture Notes in Computer Science, pp. 352-361, Springer-Verlag, Nov. 2006.

[9] R. C.-W. Phan, "Cryptanalysis of a New Ultralightweight RFID Authentication Protocol - SASI," IEEE Trans. Dependable Sec. Comput., vol. 99, no. 1, 2008.

[10] A. Sadighian and R. Jalili, "Afmap: Anonymous forward-secure mutual authentication protocols for rfid systems," The Third IEEE International Conference on Emerging Security Information, Systems and Technologies (SECURWARE 2009), pp. 31-36, 2009.

\section{Introduction}

One of automatic objects, people and animals identification technologies is Radio Frequency Identification (RFID) that widely developed nowadays. Three entities of an RFID system are tag, reader and back-end database. Tag is a microchip with constraint computational capabilities and storage resources which communicates with reader by an antenna. The reader is the unit which can achieve the saved information in the tags by radio frequency. Back-end database stores tag's identifiers, pseudonyms and keys that the reader uses through the tag authentication process.

One of the most concerns in RFID applications is security. Privacy, mutual authentication and untraceability are such problems that aroused researchers' interest on RFID security. For addressing theses problems a lot of protocols have been proposed $[8,4,7]$. However, most of these attempts $[6,5,1,2,3,9]$ have failed to achieve their desired security goals.

Recently, Sadighian and Jalili[10] have proposed a new protocol called Anonymous Forward-security Mutual Authentication Protocol or in short AFMAP for RFID systems. Among other security properties, they have claimed that AFMAP has sufficient resistance against desynchronization attack. However, in this paper, we show that the AFMAP protocol is vulnerable to the desynchronization attack.

The reminder of this paper is organized as follows: section 2 describes AFMAP. Our desynchronization attack is presented in section 3. Section 4 concludes the paper.

\section{Description of the AFMAP protocol}

It is an accepted assumption to consider the communication channel between reader and back-end database secure. Hence, throughout this paper we do not distinguish between back-end database and reader and just call them reader.

The AFMAP protocol is a mutual authentication protocol based on a secure hash function denoted by $h$. Tag and reader in the AFMAP have only one shared value which is denoted by $k$ and updated after each successful run 
of protocol. In round $i$ this secret key denoted by $k_{i}$. To resist desynchronization attack both tag and reader keep two records of key, i.e. $k_{\text {ilast }}$ and $k_{i}$, where $k_{\text {ilast }}$ denotes the previous value of $k_{i}$. Several time related parameters are used in the AFMAP protocol. These parameters are denoted by $t_{\text {sys }}, t_{\text {tag }}, t_{\max }$ and $t_{0}$ and defined as follows:

$t_{\text {sys }}$ : The system's time is a parameter that reader indicates the system time by it.

$t_{t a g}$ : The tag's time is a parameter that is initiated by $t_{0}$ and updates after each successful run of protocol.

$t_{\text {max }}$ : The maximum time is a predefined constant parameter that tag considers it as the upper bound of system's time.

$t_{0}$ : It is a parameter that initiates $t_{t a g}$.

The details of AFMAP protocol is depicted in Algorithm 1. We have used the following assumptions and notations in the description of algorithm:

- All parameters in the protocol are of length $t$-bit.

- The expression $A \rightarrow B$ refers to assigning $A$ to $B$.

- For a finite set $\mathcal{X}, x \stackrel{\$}{\leftarrow}$ is the experiment of uniformly choosing a random element from $\mathcal{X}$ and assigning it to $x$.

In the next section we analyze the security of AFMAP.

\section{Desynchronization Attack against AFMAP}

Our desynchronization attack is based on this observation that an attacker can modify both $t_{\text {sys }}$ and $n_{1}$ such that the tag authenticates the reader. On the other hand, if $t_{\text {sys }} \leq t_{\text {tag }}$ or $t_{\text {sys }} \geq t_{\max }$ the tag does not authenticate the reader. So, if the attacker can force the tag to set $t_{\text {tag }}$ to a value almost equal to $t_{\text {max }}$, tag does not authenticate the reader any more, because $t_{\text {sys }} \leq t_{\text {tag }}$. So, the attacker needs to determine an upper bound for $t_{\max }$. Hence, at the first step we explain how the attacker can extract the value of $t_{\max }$. Algorithm 2 presents an approach to determine the accurate value of $t_{\max }$ in AFMAP. In this algorithm the following notations are used:

- Given finite set $\mathcal{X},|\mathcal{X}|$ denotes the cardinality of $\mathcal{X}$.

- $A^{i}$ denotes the $i^{\text {th }}$ bit of $A$. More precisely $A^{0}$ and $A^{|A|-1}$ denote the least significant bit and most significant bit of $A$ respectively.

Line 3 of Algorithm 2 guarantee that $t_{\text {sys }}^{\prime}>t_{\text {tag }}$. On the other hand, $X^{\prime}=h\left(k_{i} \oplus\left(n_{1} \oplus t_{\text {sys }}^{\prime} \oplus t_{\text {sys }}\right) \oplus t_{\text {sys }}^{\prime}\right)=h\left(k_{i} \oplus n_{1} \oplus t_{\text {sys }}\right)=X$. Hence, the tag authenticates the reader if and only if $t_{\text {sys }}^{\prime}=t_{\max }^{\prime}<t_{\max }$. Otherwise the tag sends two pseudo random numbers. In this case the reader does not authenticate the tag and the attacker, through the condition of line 11, will find out that $t_{\max }<t_{\text {max }}^{\prime}$ and in the next attempt it selects a smaller value for $t_{\text {max }}^{\prime}$, line 13. Otherwise, in the next attempt the attacker selects a bigger value for $t_{\text {max }}^{\prime}$. The attacker repeats this game at most $\left|t_{\text {max }}\right|$-time to achieve 


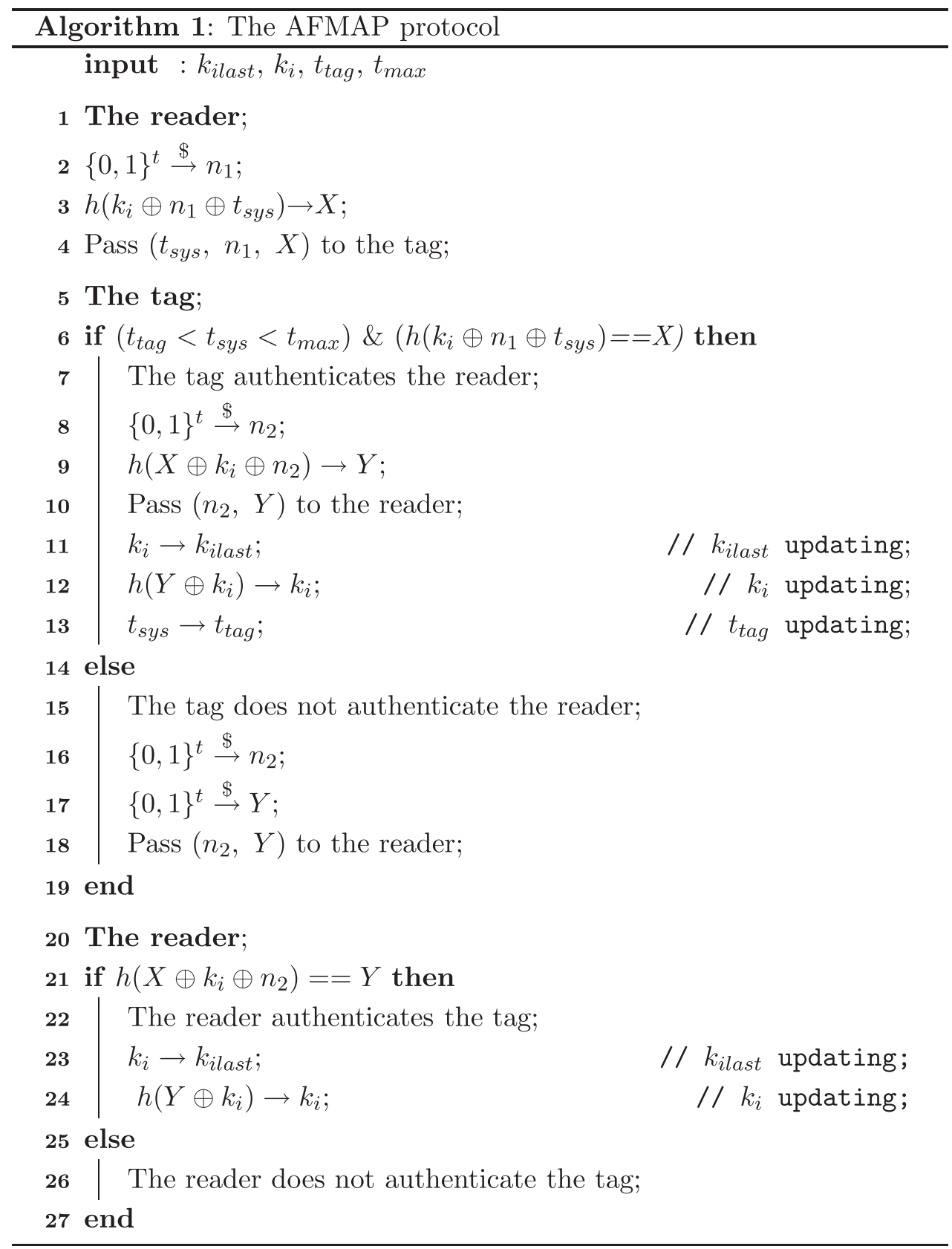

the accurate value of $t_{\max }$. Hence, the attacker can retrieve the tag's $t_{\max }$ of AFMAP, line 18.

However, when Algorithm 2 is terminated, the tag has assigned $t_{\max }-1$ to $t_{\text {tag }}$. It means that for the later runs of protocol and for any $t_{\text {sys }}$ that the reader passes to the tag, the condition $t_{\text {sys }}<t_{\text {tag }}$, line 6 of Algorithm 1 , would not be satisfied. Hence, the tag never authenticates the reader any more and we can claim that the tag and the reader were desynchronized. The expected complexity of attack is $\left|t_{\max }\right|$ and the success probability is 1 .

remark 1 . One may argues that any value which is returned by the tag is meaningless for the attacker, because it does not know the secret key $k_{i}$ and therefore the expected value for $Y$. However, if the tag returns random values, happens if $t_{\max }^{\prime}>t_{\max }$, then the reader does not authenticate the tag and it 


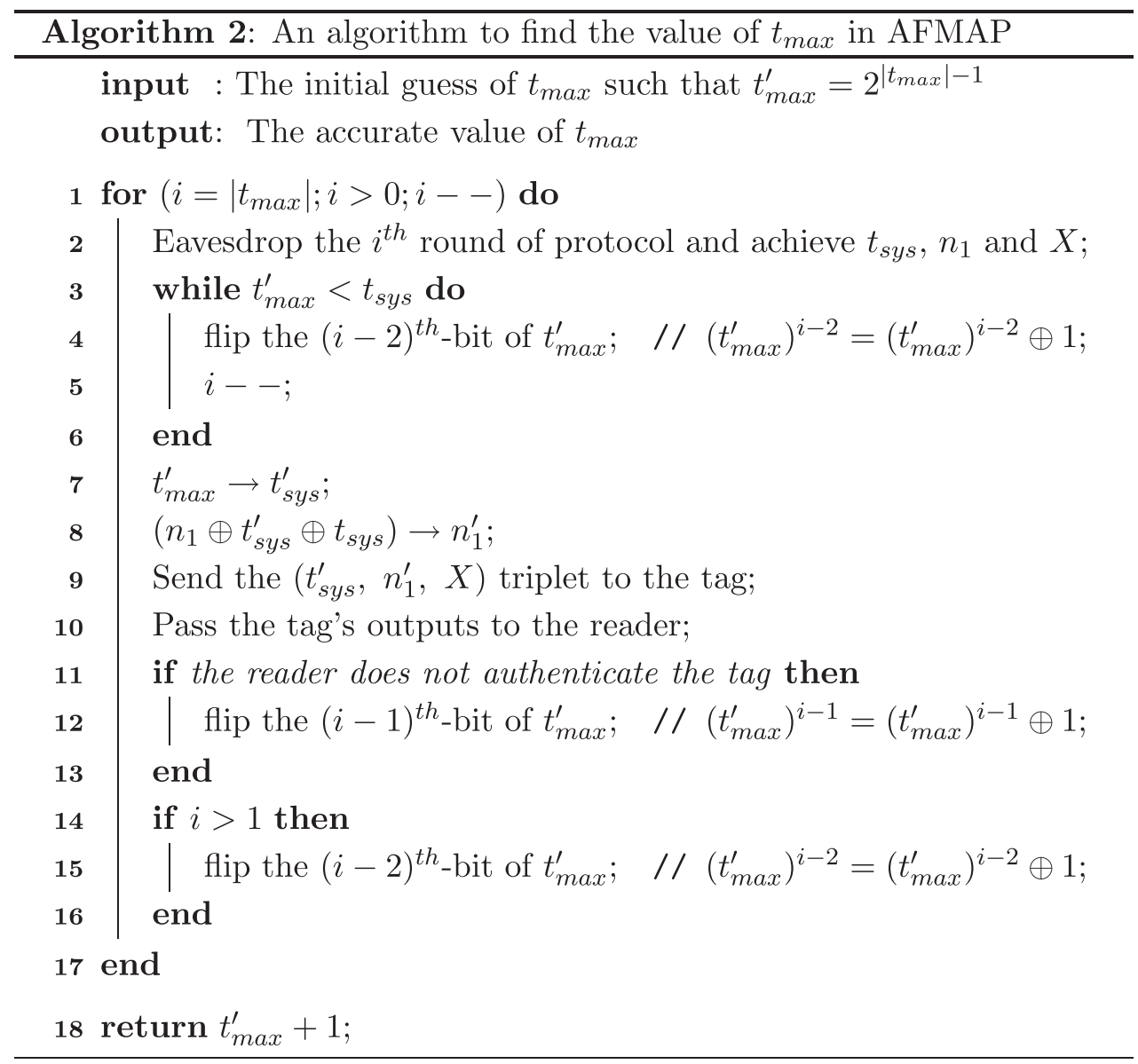

is expected to restart the game. It can be evidence that the attacker uses to validate its guesses. On the other hand, if the tag authenticates the reader, which is equivalent to the case $t_{\max }^{\prime}<t_{\max }$, the attacker must wait for another game running by the reader. By the way, we put this assumption on the protocol that the attacker has the capability to find out whether the reader has authenticated the tag. This is not an unrealistic assumption. Recall that, in practical for many applications, we expect a physical observation after the tag authentication process, e.g. a door would be opened, we receive some information related to object, and so on.

remark 2 . To overcome this attack, both reader and tag should consider $t_{\text {sys }}$ in their calculation such that they are not authenticate each other if the attacker changes $t_{\text {sys. }}$. A potential solution is to change the calculation of $X$ and $Y$ as follows:

$$
\begin{gathered}
X=h\left(h\left(k_{i} \oplus n_{1}\right) \oplus t_{\text {sys }}\right) \\
Y=h\left(X \oplus h\left(k_{i} \oplus t_{\text {sys }}\right) \oplus n_{2}\right)
\end{gathered}
$$

Since, the attacker has no knowledge about $k_{i}$ and it can not determine $h\left(k_{i} \oplus\right.$ $\left.t_{\text {sys }}\right)$ or $h\left(k_{i} \oplus n_{1}\right)$ any change in $t_{\text {sys }}$ leads to a situation that protocol does not run properly and tag does not authenticate the reader or vise versa. However, the cost of this security strengthening is two extra calls to the hash function for both tag and reader. 


\section{Conclusion}

In this paper we analyzed the security of Anonymous Forward-Secure Mutual Authentication Protocol (AFMAP). We presented a desynchronization attack against this protocol with small complexity and the success probability of 1 . In addition, we proposed a modification to the protocol which prevents the presented attack. However, it costs two extra calls to the hash function. It would be interesting to study the security of the modified protocol.

\section{Acknowledgments}

The authors would like to thank Dr. Praveen Gauravaram for helpful comments. 